Aggregation of Partial Rankings - An Approach Based on the Kemeny Ranking Problem

Peer-reviewed author version

NAPOLES RUIZ, Gonzalo; DIKOPOULOU, Zoumpolia; PAPAGEORGIOU, Elpiniki; Bello, Rafael \& VANHOOF, Koen (2015) Aggregation of Partial Rankings - An Approach Based on the Kemeny Ranking Problem. In: Rojas, Ignacio; Joya, Gonzalo.; Catala, Andreu (Ed.). Advances in Computational Intelligence, p. 343-355.

DOI: 10.1007/978-3-319-19222-2_29

Handle: http://hdl.handle.net/1942/19809 


\title{
Aggregation of partial rankings - an approach based on the Kemeny ranking problem
}

\author{
Gonzalo Nápoles ${ }^{1,2, *}$, Zoumpoulia Dikopoulou², Elpiniki Papageorgiou³, \\ Rafael Bello ${ }^{1}$ and Koen Vanhoof ${ }^{2}$ \\ ${ }^{1}$ Universidad Central "Marta Abreu" de Las Villas, Santa Clara, Cuba \\ ${ }^{2}$ Hasselt University, Diepenbeek, Belgium \\ ${ }^{3}$ Technological Education Institute of Central Greece, Lamia, Greece \\ *gnapoles@uclv.edu.cu
}

\begin{abstract}
Aggregating the preference of multiple experts is a very old problem which remains without an absolute solution. This assertion is supported by the Arrow's theorem: there is no aggregation method that simultaneously satisfies three fairness criteria (non-dictatorship, independence of irrelevant alternatives and Pareto efficiency). However, it is possible to find a solution having minimal distance to the consensus, although it involves a NP-hard problem even for only a few experts. This paper presents a model based on Ant Colony Optimization for facing this problem when input data are incomplete. It means that our model should build a complete ordering from partial rankings. Besides, we introduce a measure to determine the distance between items. It provides a more complete picture of the aggregated solution. In order to illustrate our contributions we use a real problem concerning Employer Branding issues in Belgium.
\end{abstract}

Keywords: Partial Rankings, Aggregation, Ant Colony Optimization.

\section{Introduction}

The aggregation of multiple preferences has been widely studied by economists under social choice theory. In recent years reasoning based on permutations has gained great attention due to the applications in solving decision-making problems. For example, a main aspect in some machine learning tasks is how to combine the output of multiple classifiers, in order to determine the most adequate class. Other relevant applications include: computational biology [1], multi-agent planning [2], voting in elections [3], information retrieval [4], among others interesting fields.

Formally the aggregation of preferences could be summarized as follows: given $N$ orderings over $M$ objects/items where each ranking denotes the preference of a single expert, then the goal is to build a consensus (aggregated) ranking taking into account all input rankings. Borda [5] and Condorcet [6] proposed diverse ways of aggregating the preferences of the multiple voters, and argued over which method is the right one. Unfortunately, Arrow [7] proved that there is no right approach, since there exists no aggregation method that concurrently satisfies three fairness criteria: non-dictatorship, Pareto efficiency and independence of irrelevant alternatives. 
Despite this negative result, it is possible to compute an aggregated ranking having minimal distance to the global consensus. This ranking/ordering is also known as the Kemeny ranking. In [8] the authors performed an extensive study including different methods (e.g. Branch and Bound, approximate algorithms) for computing the Kemeny ranking. It was also concluded that heuristic approaches are recommended in contexts having weak or no consensus. More recently, Aledo at al. [9] introduced an approach based on evolutionary computation, which clearly outperformed the remaining tested algorithms. However, this model is based on the Kendall distance and hence it cannot be directly applied to the aggregation of incomplete preferences.

Inspired on this work we introduce an approximate model for aggregating partial rankings which uses Ant Colony Optimization (ACO) as optimizer. The main reason behind this decision is moved by the strong ability of ACO for solving combinatorial problems [10] and also by its scheme for generating new states. In a few words, ACO exploits the heuristic information for improving the search, and this knowledge could be easily computed from input data. Moreover, we present a measure for computing the relative distance between two items/objects in the final ranking (which is entirely based on the induced deviation to the experts' consensus).

The rest of the paper is organized as follows. The next section makes an overview about the Kemeny ranking problem and describes an extension for aggregating partial rankings. It includes the design of the objective function to be optimized, and also the measure for computing the distance between two objects. Section 3 reviews the main ideas of ACO-based optimizers, which will be used next for generating the candidate ranking. In Section 4 we explain how to estimate the heuristic information from input data for two different scenarios. Section 5 presents a real study case about employees' preferences in Belgium when they look for an employer. In the last section the authors discuss conclusions and future research directions.

\section{Extending the Kemeny ranking problem}

Solving the Kemeny ranking problem is equivalent to compute the consensus ranking for a set of input rankings. The reader can find the formulation of this problem in [11] although it could be summarized as follows: given a set of $N$ rankings $\pi_{1}, \pi_{2}, \ldots, \pi_{N}$ with $M$ elements, the Kemeny ranking problem consists on finding the ranking $\pi_{*}$ that satisfies the expression (1). Here $\pi_{*}$ is the central permutation to be computed by the model, whereas $d_{1}\left(\pi, \pi_{*}\right)$ denotes the Kendall distance [12]. In brief, the Kendall-Tau distance between two rankings $\pi_{1}$ and $\pi_{2}$ is defined as the total number of items pairs over which they disagree. However, this function assumes that all objects are ranked where ties are no allowed, hence the Kendall-Tau distance is not a suitable alternative when we want to face the aggregation of partial rankings.

$$
\pi_{*}=\operatorname{argmin}_{\pi_{i}} \frac{1}{N} \sum_{i=1}^{N} d_{1}\left(\pi_{i}, \pi_{*}\right)
$$


In many important applications, rankings are only partial, in the sense that ties are allowed. This happens, for example, when only the top, say $K$, elements are ordered, while all the remaining elements are assumed to have rank $K+1$ [13]. It is possible a second scenario where each expert $X_{i}$ selects $K_{i}$ items as relevant, leading to a ranking with $\left(M-K_{i}\right)$ elements are tied at the $K_{i}+1$ position. To deal with such scenarios we need to extend the Kemeny ranking problem by replacing the Kendall-Tau distance by a measure able of comparing partial rankings. In this paper we adopt the Hausdorff distance [14] since it is based on the Kendall-Tau principle. Besides, this distance has been widely studied and shown to have especially nice mathematical and algorithmic properties, particularly with regard to rank aggregation [15].

$$
d_{2}\left(\pi_{i}, \pi_{*}\right)=\left|\mathcal{D}\left(\pi_{i}, \pi_{*}\right)\right|+\max \left\{\left|\mathcal{R}_{1}\left(\pi_{i}, \pi_{*}\right)\right|,\left|\mathcal{R}_{2}\left(\pi_{i}, \pi_{*}\right)\right|\right\}
$$

In the equation (2) the set $\mathcal{D}\left(\pi_{i}, \pi_{*}\right)$ denotes all items pairs that appear in different order, $\mathcal{R}_{1}\left(\pi_{i}, \pi_{*}\right)$ represents the set of all objects pairs which are tied in $\pi_{i}$ but not tied in the ordering $\pi_{*}$, whereas $\mathcal{R}_{2}\left(\pi_{i}, \pi_{*}\right)$ is the set of elements pairs which are tied in $\pi_{*}$ but not tied in the ranking $\pi_{i}$. However, if we consider that the ranking $\pi_{*}$ is complete then the expression ( 2 ) could be notably reduced as follows:

$$
d_{2}\left(\pi_{i}, \pi_{*}\right)=\left|\mathcal{D}\left(\pi_{i}, \pi_{*}\right)\right|+\left(\begin{array}{c}
M-K_{i} \\
2
\end{array}\right)
$$

In other words, $\left|\mathcal{R}_{2}\left(\pi_{i}, \pi_{*}\right)\right|=0$ since $\pi_{*}$ is a complete ordering (i.e. the candidate solution to be generated by the selected optimizer). Likewise, the reader could observe that $\left|\mathcal{R}_{1}\left(\pi_{i}, \pi_{*}\right)\right|=\left(\begin{array}{c}M-K_{i} \\ 2\end{array}\right)$ where $K_{i}$ is the number of relevant items, according to the $i$ th respondent. Next equation shows the normalized objective function, which should be minimized during the search process. The closer to zero the evaluation, the closer the permutation $\pi$ to the consensus. In the next Section we describe the main ideas of ACO-based algorithms, but first we present a new measure for computing the relative distance between two consecutive objects in a consensus ranking.

$$
H(\pi)=\frac{1}{N} \sum_{i=1}^{N} \frac{\left|\mathcal{D}\left(\pi_{i}, \pi\right)\right|+\left(\begin{array}{c}
M-K_{i} \\
2
\end{array}\right)}{M(M-1) / 2}
$$

\subsection{Measuring the distance between two objects}

As discussed, the goal of the Kemeny ranking problem is to find a complete ordering from a set of input rankings, having minimal distance to the consensus. In this scheme the inputs could be partial or complete. The aggregated ranking is a suitable tool when we want to face decision-making problems based on permutations, but sometimes this knowledge is not enough, and further analysis is often required. For instance, a central question when experts interpret a consensus ordering is: which is the relative distance between two consecutive objects? Next we introduce a strategy to solve this question, which is completely based on the objective function. 
Let us consider a consensus ordering $\pi=\{\pi(1), \pi(2), \ldots, \pi(l), \pi(l+1), \ldots, \pi(M)\}$ that minimizes (4) such as $x=\pi(l)$ and $y=\pi(l+1)$. In other words, $\pi$ is a solution for the Kemeny ranking problem where $x<y$. The equation (5) displays the induced deviation $\delta(y, x)_{\pi}$ which measures the distance between such items.

$$
\delta(y, x)_{\pi}=\frac{\left|H(\pi)-H\left(\check{\pi}_{y<x}\right)\right|}{\sum_{k}\left|H(\pi)-H\left(\check{\pi}_{\pi(k+1)<\pi(k)}\right)\right|}
$$

In this equation $\check{\pi}_{y<x}$ represents a ranking obtained from $\pi$ where objects $x$ and $y$ were exchanged, whereas $k=1, \ldots, M$ indexes the elements. The reader could notice that $\delta(y, x)_{\pi}=0$ if $\pi_{x<y}$ and $\check{\pi}_{y<x}$ have the same heuristic value, and accordingly we can conclude that $x$ and $y$ are tied (i.e. they are at the same level). It should be stated that our model attempts to compute a complete ordering using a set of partial and/or complete rankings as input data. However, it is possible to obtain a solution implicitly having tied items. Even it is possible that $\sum\left|H(\pi)-H\left(\check{\pi}_{\pi(k+1)<\pi(k)}\right)\right|=0, \forall k$, so we must assume that $\delta(y, x)=0$ for all pairs of elements.

The central idea behind this measure could be summarized as follows: which is the induced deviation to the consensus if $x$ and $y$ are exchanged? In principle the ordering should have minimal distance to the general consensus, otherwise the results could be confusing. However, as was discussed before, finding this ranking involves a complex combinatorial problem for only a few experts and alternatives. That is why we prefer to adopt a heuristic approach based on Swam Intelligence.

\section{Ant Colony Optimization}

A central component in the proposed model is the generation of feasible permutations using the objective function for guiding the search. In this paper we use ACO methods as optimizers, where each permutation comprises a possible solution.

The ACO metaheuristic is a search method for addressing combinatorial problems, which is inspired on a colony of agents (ants) [16]. Real ants in nature search for food in a random proximity to the nest. Once the ants found a source of food, they evaluate this source according to quality and quantity. In the path back to the nest, they deposit a chemical pheromone trail on the ground, in order to guide the rest of the colony to the food source [17]. Therefore, ACO is a fully constructive model where each ant builds a candidate solution of the problem by exploring a construction graph.

Each artificial ant moves from one state to another during the search process (here states are components of the solution). The preference of moving from one node to the other mainly depends on two values associated to each connection:

- The artificial information $\tau_{i j}$ is directly based on the pheromone trails, and it is iteratively updated by ants during the algorithm progress.

- The heuristic information $\eta_{i j}$ denotes the preference of moving from one state to another. It should be specified that this knowledge is not modified during the algorithm execution, so it must be carefully estimated. 
From the perspective of the Kemeny ranking problem, the equation (6) denotes the probability of accepting the $j$ th state (i.e. elements to be ordered) at the $i$ th position of the candidate ranking, $\mathcal{N}_{i}^{k}$ is the set of unvisited states for the $k$ th ant, while $\alpha$ and $\beta$ are two parameters which are used for controlling the strength of the pheromone trails and the heuristic information over the decision, respectively.

$$
P_{i j}^{k}(t+1)=\frac{\left[\tau_{i j}(t)\right]^{\alpha}\left[\eta_{i j}\right]^{\beta}}{\sum_{r \in \mathcal{N}_{i}^{k}}\left[\tau_{i r}(t)\right]^{\alpha}\left[\eta_{i r}\right]^{\beta}}, j \in \mathcal{N}_{i}^{k}
$$

After the construction process is complete, it is necessary to update all pheromone trails using the solutions found by agents. As a first stage, pheromone evaporation takes place uniformly reducing all pheromone trails. Subsequently, one or more solutions are used to increase the value of such paths included in selected solutions. It is a sensible issue in ACO-based algorithms. Essentially, most of ACO variants mainly differ in the strategy for updating the pheromone trail at each cycle.

\subsection{Ant System}

The Ant System (AS) was the first ACO algorithm [18]. In AS the pheromone trails is updated once all ants have completed their tours. As a first step all pheromone trails are uniformly evaporated using a constant factor $0<\rho<1$. After that, each ant $k$ deposits a quantity of pheromone $\Delta \tau_{i j}$ on those connections that belong to its solution. It should be mentioned that the value $\Delta \tau_{i j}$ is calculated according to the quality of the solution found by the $k$ th ant. The following equation shows both procedures, where $\rho$ denotes the evaporation rate, whereas $P$ is the number of agents.

$$
\tau_{i j}(t+1)=(1-\rho) \tau_{i j}(t)+\sum_{k=1}^{P} \Delta \tau_{i j}^{k}
$$

On arcs which are not regularly chosen by ants, the associated pheromone strength will decrease exponentially with the number of iterations, whereas arcs often chosen by agents will receive more pheromone and therefore they are more likely to be chosen in future cycles. However, deeper simulations reported in [18] proved that better results could be computed if only the global-best solution is used for updating the pheromone trails, instead of using all individuals belonging to the swarm.

\subsection{Ant Colony System}

The Ant Colony System (ACS) improves the AS method by exploiting the global-best solutions found by ants during the search stage [19]. As result, the algorithm enhances the exploitation features of ants when they build a solution, instead of exploring new areas of the solution space. This goal is achieved using three mechanism: a strong elitist strategy for updating pheromone trails, a rule for updating pheromone trails during the search phase, and a pseudo-random rule when selecting new states. 
The following equation formalizes the strategy when updating the pheromone trails, where $\tau_{i j}^{*}$ denotes the pheromone quantity associated to the agent having better heuristic value. It means that the evaporation step takes place in all arcs, but the updating process only occurs in the tour traveled by the best individual.

$$
\tau_{i j}(t+1)=(1-\rho) \tau_{i j}(t)+\rho \tau_{i j}^{*}(t)
$$

In order to fully exploit the best knowledge discover by ants, ACS also introduces a pseudo-random proportional rule (see next equation). More specifically, if a random number $q \sim U(0,1)$ falls below $q_{0}$ then the agent will move to the state maximizing the product between pheromone trail and heuristic information, otherwise ACS will adopt the standard decision rule (6). The value $q_{0}$ is a parameter that should be fixed by the expert; when it is close to 1 , exploitation is favored over exploration.

$$
j=\underset{r \in \mathcal{N}_{i}^{k}}{\operatorname{argmax}}\left\{\left[\tau_{i j}(t)\right]^{\alpha}\left[\eta_{i j}\right]^{\beta}\right\} \text { if } q \leq q_{0}
$$

Finally, in the ACS model ants use a further rule for updating the pheromone trails whey they are building the candidate solution (see next equation). This approach has the same effect of decreasing the probability of selecting the same path for all ants, as a way of introducing a balance between exploitation and exploration.

$$
\tau_{i j}(t+1)=(1-\rho) \tau_{i j}(t)+\rho \tau_{i j}(0)
$$

The ACS algorithm regularly computes better solutions regarding the AS, since we know that ACO-based model performs better if artificial ants exploit the best solution found during the search process. In the next sub-section we revise another variant that adopt a similar principle attempting improving the performance.

\subsection{MAX-MIN Ant System}

The MAX-MIN Ant System (MMAS) was specifically developed to achieve stronger exploitation of solutions, avoiding stagnation states [20]. In a nutshell, we could define a stagnation state as the situation where ants construct the same solution over and over again and the exploration stops. This model has the following features.

Equally to the ACS, a strong elitist strategy regulates the agent which is allowed to update the pheromone trails. It could be the ant having better evaluation so far, or the agent with the best tour in the current iteration. Second, all pheromone trails are limited in the range $\left[\tau_{M I N}, \tau_{M A X}\right]$. If $\tau_{M I N}>0$ for all solution components, then the probability of choosing a specific state will never be zero, which avoids stagnation configurations. As a final point, pheromone trails are initialized with $\tau_{M A X}$ to ensure further exploration of the search space at the beginning of the optimization phase. 


\section{Estimating the heuristic information}

Another crucial component when solving combinatorial problems using an ACO-based algorithms is the estimation of the heuristic matrix. During the search process ants use this information to guide their movements (i.e. selection of a new state when they are building the candidate solution). If this matrix is appropriately estimated, then the ACO metaheuristic will lead to high-quality solutions, otherwise the algorithm will produce sub-optimal rankings. Next we explain two strategies to estimate this component from input data, assuming two partial rankings aggregation scenarios.

\subsection{Aggregation of multiple top- $K$ rankings}

The first scenario takes place when each expert (hereinafter called respondent) selects the top- $K$ objects (hereinafter called factors). It means that each input rankings will be partial in the sense that only the top- $K$ factors are ordered, whereas the other $(M-K)$ factors are tied at the $K+1$ position. The reader can notice that estimating the matrix for the first $K$ factors across the $M$ positions is equivalent of computing the total number of times that the $j$ th factor was observed in the $i$ th position. For the remaining $(M-K)$ places it cannot be directly used since these factors are tied, however, we could count how many times a factor was not included into the top- $K$. Next equation formalizes this reasoning, assuming $N$ as the total number of respondents.

$$
\eta_{i j}= \begin{cases}X_{i j} / N, & i \leq K \\ Y_{j} /(N(M-K)), & i>K\end{cases}
$$

Example\#1. Let us consider a ranking aggregation problem with 5 possible factors and 5 respondents, where each expert selected the top-3 factors. Next table shows this scenario. Observe that each row (respondent) comprises a partial ordering where factors are associated to specific positions. According to (11) the heuristic value of accepting the second factor at the first ranking position is $\eta_{12}=2 / 5$.

Table 1. Example of a dataset when aggregating multiple top- $K$ rankings.

\begin{tabular}{|l|ccccc|}
\hline & $F_{1}$ & $F_{2}$ & $F_{3}$ & $F_{4}$ & $F_{5}$ \\
\hline$R_{1}$ & 1 & 2 & $K+1$ & 3 & $K+1$ \\
$R_{2}$ & 2 & 1 & $K+1$ & $K+1$ & 3 \\
$R_{3}$ & 1 & 3 & $K+1$ & $K+1$ & 2 \\
$R_{4}$ & 1 & 3 & $K+1$ & 2 & $K+1$ \\
$R_{5}$ & 2 & 1 & 3 & $K+1$ & $K+1$ \\
\hline
\end{tabular}

Note that $\eta_{52}=0$ since $F_{2}$ was always included into the top- 3 sites and according to the equation (6) the probability $P_{52}$ will be zero! However, this probability should not be zero because it is still possible building a solution having $F_{2}$ at the last position. That is why we replace all zero-values by $\eta_{M I N}=\min \left\{\eta_{i j}\right\}$ such as $\eta_{i j} \neq 0$, so we guarantee that all states could be visited by ants when they are building a solution. 


\subsection{Aggregation of multiple top- $K_{l}$ rankings}

This scenario is more complex since each respondent is free of selecting $K_{l}$ factors such as $1 \leq K_{l} \leq M$. Since the number of related factors is not fixed, we cannot simply count the number of times that a factor was observed at each site, we also must quantify the number of times that the factor could be observed at each position. It allows computing a more realistic heuristic matrix. Next equation summarizes this idea, where $X_{i j}$ denotes the number of times the $j$ th factor was observed at the $i$ th position, $Q_{j}$ is the set of input rankings where the $j$ th factor was not included into the top- $K_{l}$ positions, $D_{k}$ represents the set of feasible ranking positions (i.e. they do not induce new tied elements) for the $j$ th factor, while $E_{k i}(j)$ is a binary function. This function responds a simple question: could be the $j$ th factor assigned to the $i$ th ranking position?

$$
\eta_{i j}=\frac{1}{N}\left(X_{i j}+\sum_{k \in Q_{j}} \frac{E_{k}(i)}{\left|D_{k}\right|}\right)
$$

Example\#2. Let us consider a ranking aggregation problem with 5 possible factors and 5 respondents, where each expert $R_{l}$ selected the top- $K_{l}$ factors, as summarizes the next table. According to (12) the heuristic value of accepting $F_{2}$ at the first position is $\eta_{12}=1 / 5(2+0 / 2+0 / 3)=2 / 5$ because $Q_{2}=\left\{R_{1}, R_{4}\right\}, D_{1}=\{4,5\}, D_{4}=\{3,4,5\}$ and $E_{k}(i)=0, \forall i \in\{1,4\}$ since $1 \notin\left(D_{1} \cup D_{4}\right)$. It means that $F_{2}$ cannot be assigned to the first position, without introducing a new tied element. It should be also mentioned that tied factors into the top- $K_{l}$ relevant positions are not allowed.

Table 2. Example of a dataset when aggregating multiple top- $K_{l}$ rankings.

\begin{tabular}{|l|ccccc|}
\hline & $F_{1}$ & $F_{2}$ & $F_{3}$ & $F_{4}$ & $F_{5}$ \\
\hline$R_{1}$ & 1 & $K_{1}+1$ & 2 & $K_{1}+1$ & 3 \\
$R_{2}$ & 3 & 1 & $K_{2}+1$ & 2 & $K_{2}+1$ \\
$R_{3}$ & 2 & 1 & 4 & 5 & 3 \\
$R_{4}$ & 2 & $K_{4}+1$ & $K_{4}+1$ & $K_{4}+1$ & 1 \\
$R_{5}$ & 5 & 3 & 1 & 2 & 4 \\
\hline
\end{tabular}

Similarly to the above scenario, we must avoid zero-values in the heuristic matrix, although this situation is possible (i.e. the factor was never observed in a position and there is no chance to be observed without inducing a tied element). However, it is still possible to build a candidate solution with this feature having minimal distance to the general consensus, therefore it must be considered as well. In such cases the probability should not be zero but low (e.g. $\eta_{M I N}=\min \left\{\eta_{i j}\right\}$ such as $\eta_{i j} \neq 0$ ).

Notice that other scenarios are possible when aggregating partial rankings (e.g. tied elements in the top- $K$ are allowed). In such cases our methodology is still useful since the modeling (i.e. generation of permutations) could be adopted, but some changes are required. In the following section, we explore the performance of our methodology in a real study case concerning Employer Branding issues in Belgium. 


\section{Numerical simulations}

Which are the most significant factors to be considered by employees when they look for an employer? The answer for this question embraces a valuable knowledge for any company since it provides the key for attracting the best employees, hence being more competitive. In this section we address this complex issue by solving a partial ranking aggregation problem. It includes two different scenarios:

a) Each respondent $R_{l}$ selects the top-5 factors from $M=17$ possible factors.

b) Each respondent $R_{l}$ is free of selecting the most significant $K_{l}$ factors (there is no limit regarding the number of relevant factors, but $1 \leq K_{l} \leq 17$ ).

In this study 14.585 respondents (aged between 18 and 65 years old) from Belgium where consulted ${ }^{1}$. As mentioned before, in the survey they evaluated 17 global factors elaborated by marketing experts, which are listed in the following table:

Table 3. Global factors evaluated by each respondent during the online survey.

\begin{tabular}{|l|l|}
\hline$F_{1}$ & Financially sound \\
$F_{2}$ & Offers quality training \\
$F_{3}$ & Offers long-term job security \\
$F_{4}$ & Offers international / global career \\
$F_{5}$ & Future prospects / career opportunities \\
$F_{6}$ & Strong management \\
$F_{7}$ & Offers interesting jobs (job description) \\
$F_{8}$ & Pleasant working environment \\
$F_{9}$ & Competitive salary package \\
$F_{10}$ & Good balance between life and work \\
$F_{11}$ & Well located \\
$F_{12}$ & Strong image / pursues strong values \\
$F_{13}$ & Quality products / services offered \\
$F_{14}$ & Deliberately handles the environment and society \\
$F_{15}$ & Uses the latest technologies / innovative \\
$F_{16}$ & Provides flexible working conditions \\
$F_{17}$ & Encourages diversity (age, gender, ethnicity) \\
\hline
\end{tabular}

Besides, respondents are grouped according to the sector that they belong, resulting the following categories: automotive, business services, chemical and pharmaceutical industry, construction, education/government/care, energy services, human resources, informatics-consultancy, retail, travel/leisure/hospitality, industry and manufacturing, finance, food, transportation and logistics, and other.

\footnotetext{
${ }^{1}$ Randstad was founded in 1960 by Frits Goldschmeding in the Netherlands. This company plays a pivotal role in the World of Work since it expanded its operations to 39 countries, representing more than 90 percent of the global HR services market. Actually, Randstad company is now the second largest HR services provider in the world. See http://www.randstad.com.
} 
For ACO-based methods we adopt the following parameters: $\alpha=3$ and $\beta=2$ since the knowledge learned by ants is often more confident, the evaporation constant is set as $\rho=0.6$, whereas the pheromone matrix is initialized with $\tau_{i j}(0)=0.5$. In the case of the ACS method, we fix $q_{0}=0.7$; whereas the pheromone limits $\lambda_{M I N}$ and $\lambda_{M A X}$ in the MMAS algorithm are computed as suggested [19]. Finally, we use a swarm having 17 artificial ants (one ant per factor) and 150 generations.

\subsection{First scenario: each respondent $R_{l}$ selects the top- $K$ factors}

The first experiment consists on finding the best optimizer. With this purpose in mind we averaged the best heuristic value $H\left(\pi_{*}\right)$ over 10 independent trials for each sector, since our model is non-deterministic. Next figure shows the mean ranks achieved for each algorithm according to the Friedman test [21]. Using a significance level of 0.05 , corresponding to the $95 \%$ confidence interval, the Friedman test suggests rejecting the null hypothesis $\mathrm{H}_{0}(\mathrm{p}$-value $=0.0<0.05)$. As a result, we can conclude that there exist highly significant differences between at least two algorithms.

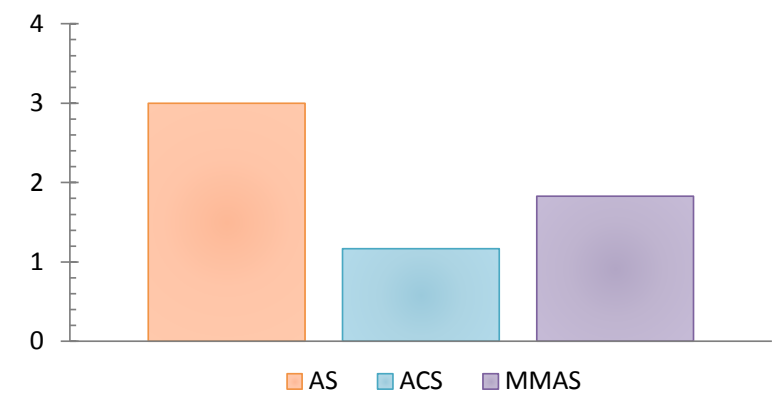

Fig. 1. Mean ranks achieved by the Friedman test for the first problem.

However, we cannot ensure that ACS is the best optimizer (notice that ACS has the lowest mean rank). As second step it is necessary to proof that ACS is involved in the highly significant differences reported by the Friedman test. To do that, we compute the Wilcoxon signed rank test [22] with the purpose of identifying significant difference between pairs of algorithms. The Wilcoxon test attempts to answer a question: do two samples represent two different populations? It suggests rejecting the null hypothesis for all pairs ( $p$-value $<0.05$ ), assuming a significance level of 0.05 . Hence, one can conclude that ACS is able of finding solutions closest to the consensus, outperforming the other algorithms when aggregating partial rankings.

Based on this result, next we select the best solution found by the ACS model for all respondents after 10 independent trials. Table 4 shows the averaged ranking $\pi$ and also the distance between consecutive factors, according to (5). It can be noted that people in Belgium prefer interesting jobs, financially sound with competitive salary packages having pleasant working environment. However, the most desirable factor is long-term security. It is confirmed by the "distance" between the factor $F_{3}$ ranked as first and the factor $F_{7}$ ranked as second: $\delta\left(F_{7}, F_{3}\right)_{\pi}=0.27$. It should be remarked that this measure has the greatest value between all pairs of consecutive factors. 
Table 4. Best ranking for the first problem and distances between consecutive factors.

\begin{tabular}{|ccc|ccc|}
\hline Position & Factor & $\delta(y, x)_{\pi}$ & Position & Factor & $\delta(y, x)_{\pi}$ \\
\hline$\pi(1)$ & $F_{3}$ & 0.0000 & $\pi(10)$ & $F_{2}$ & 0.1048 \\
$\pi(2)$ & $F_{7}$ & 0.2717 & $\pi(11)$ & $F_{13}$ & 0.0886 \\
$\pi(3)$ & $F_{8}$ & 0.0638 & $\pi(12)$ & $F_{4}$ & 0.0026 \\
$\pi(4)$ & $F_{9}$ & 0.0419 & $\pi(13)$ & $F_{14}$ & 0.0190 \\
$\pi(5)$ & $F_{1}$ & 0.0870 & $\pi(14)$ & $F_{6}$ & 0.0159 \\
$\pi(6)$ & $F_{10}$ & 0.0962 & $\pi(15)$ & $F_{12}$ & 0.0026 \\
$\pi(7)$ & $F_{11}$ & 0.0602 & $\pi(16)$ & $F_{17}$ & 0.0012 \\
$\pi(8)$ & $F_{5}$ & 0.0090 & $\pi(17)$ & $F_{15}$ & 0.0289 \\
$\pi(9)$ & $F_{16}$ & 0.1059 & - & - & - \\
\hline
\end{tabular}

\subsection{Second scenario: each respondent $R_{l}$ selects the top- $K_{l}$ factors}

In this scenario we assume that each respondent is free of selecting the most significant $K_{l}$ factors such as $1 \leq K_{l} \leq M$. Here the same simulation design discussed in the above section is assumed. The following figure summarizes the mean ranks achieved for each algorithm according to the Friedman test [21]. Adopting a significance level of 0.05 , corresponding to the $95 \%$ confidence interval, the Friedman test suggests rejecting the hypothesis $\mathrm{H}_{0}(\mathrm{p}$-value $=0.0<0.05)$. These results show that the ACS model computes the lowest mean rank, but we must analyze all pairs of optimizers.

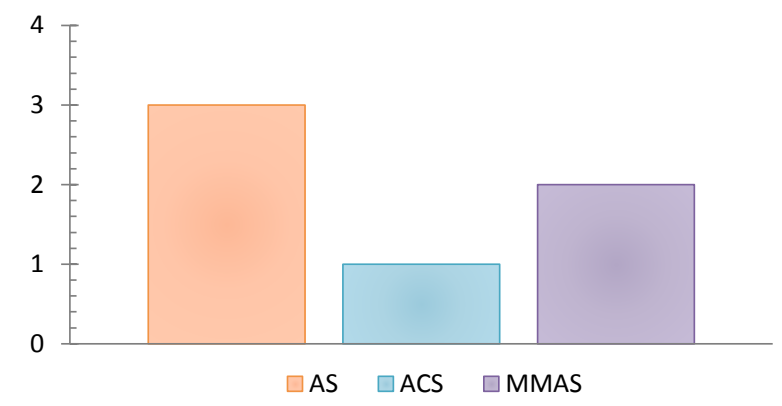

Fig. 2. Mean ranks achieved by the Friedman test for the second problem.

In order to determine that the ACS algorithm is a responsible of the highly significant differences reported by the Friedman test, next we compute the Wilcoxon signed rank test [22] for all pairs of optimizers (i.e. AS-ACS, AS-MMAS, ACS-MMAS). Assuming a significance level of 0.05 , this test proposes rejecting the null hypothesis $\mathrm{H}_{0}$ for all pairs ( $p$-value $<0.05$ ). It confirms that the ACS method is also the best variant when facing partial rankings aggregation problems with variable length.

Afterward we select the best solution found by the ACS model for all respondents after 10 independent trials. Table 4 depicts the averaged ranking $\pi$ and also the distance between consecutive factors. From these results we can surely conclude that the most desirable factor is long-term security. In addition, this aggregated ranking has the same top-5 regarding the previous one, although the order is different. 
Table 5. Best ranking for the second problem and distances between consecutive factors.

\begin{tabular}{|ccc|ccc|}
\hline Position & Factor & $\delta(y, x)_{\pi}$ & Position & Factor & $\delta(y, x)_{\pi}$ \\
\hline$\pi(1)$ & $F_{3}$ & 0.0000 & $\pi(10)$ & $F_{16}$ & 0.0128 \\
$\pi(2)$ & $F_{9}$ & 0.0852 & $\pi(11)$ & $F_{6}$ & 0.1857 \\
$\pi(3)$ & $F_{7}$ & 0.1419 & $\pi(12)$ & $F_{13}$ & 0.0349 \\
$\pi(4)$ & $F_{8}$ & 0.0171 & $\pi(13)$ & $F_{14}$ & 0.0262 \\
$\pi(5)$ & $F_{1}$ & 0.0474 & $\pi(14)$ & $F_{12}$ & 0.0615 \\
$\pi(6)$ & $F_{10}$ & 0.1410 & $\pi(15)$ & $F_{4}$ & 0.0092 \\
$\pi(7)$ & $F_{11}$ & 0.0750 & $\pi(16)$ & $F_{17}$ & 0.0072 \\
$\pi(8)$ & $F_{5}$ & 0.0009 & $\pi(17)$ & $F_{15}$ & 0.0031 \\
$\pi(9)$ & $F_{2}$ & 0.1502 & - & - & - \\
\hline
\end{tabular}

In general terms we believe that both solutions are consistent, although the second scenario is more informed (i.e. there is less tied elements) if we want to build a complete ranking. However, these solutions are not totally comparable because we use different input datasets, although we must expect some correspondence between them since the same respondent provided its best expertise for both scenarios. To overcome this issue we should formulate a "consistency" measure capable of computing the correspondence degree between both rankings (e.g. using the Hausdorff distance properties).

\section{Conclusions}

The aggregation of partial rankings could be faced using different approaches, although the absolute solution remains as an open problem. In this paper we presented a distancebased approach which directly extends the Kemeny ranking problem. In a few words, the objective is to find a permutation of factors minimizing the averaged distance to the general consensus. It involves a complex combinatorial problem for only a few experts and alternatives; that is why we prefer to use an approximate approach based on Swarm Intelligence. The proposal also includes other features such as:

- It replaces the Kendall distance by the Hausdorff distance, which allows to face partial rankings aggregation problems.

- It introduces a new measure for computing the relative distance between two consecutive items in the consensus (final) ranking.

From numerical results we concluded that the ACS method is the best variant when solving this kind of aggregation problems. It also showed that people in Belgium prefer stability (long-term security) instead of jobs financially sound. However, this outcome is not surprising and it could be a direct result of the economic crisis. As a future work we will focused on extending the simulations by including other approaches such as the Genetic Algorithm discussed in [9]. Although such experiments are absolutely required we are expecting promising results due to the ability of ACO-based methods for solving combinatorial problems, but this conjecture must be verified. 


\section{References}

1. Jackson, B.N., et al.: Consensus genetic maps as median orders from inconsistent sources. IEEE/ACM Trans. on Computational Biology and Bioinformatics. 5, 161--171 (2008)

2. Ephrati, E., Rosenschein, J.: Multi-agent planning as a dynamic search for social consensus. In: Proceedings of the 13th International Joint Conference on Artificial Intelligence, IJCAI 1993. pp. 423--429. Morgan Kaufmann. (1993)

3. Diaconis, P., et al.: A generalization of spectral analysis with application to ranked data. The Annals of Statistics. 5, 949--979 (1989)

4. Chen, H., et al.: Global models of document structure using latent permutations. In: Proc. of Human Language Technologies: The 2009 Annual Conference of the North American Chapter of the Association for Computational Linguistics, NAACL. pp. 371--379. (2009)

5. Borda, J.: Memoire sur les elections au scrutin, in: Histoire de l'Academie Royal des Sciences (1781)

6. Condorcet, M.: Sur L'application de L'analyse à la Probabilité Des Décisions Rendues à la Pluralité Des Voix, L'Imprimerie Royale, Paris (1785)

7. Arrow, K.J.: Social Choice and Individual Values. Yale University Press (1963).

8. Ali, A., Meilă, M.: Experiments with Kemeny ranking: What works when? Mathematical Social Sciences. 64, 28--40 (2012)

9. Aledo, J., Gámez, J., Molina, D.: Tackling the rank aggregation problem with evolutionary algorithms. Applied Mathematics and Computation. 222, 632--644 (2013)

10. Puris, A., Bello, R., Herrera, F.: Analysis of the efficacy of a Two-Stage methodology for ant colony optimization: Case of study with TSP and QAP. Expert Systems with Applications. 37, 5443--5453 (2010)

11. Kemeny, J.L., Snell, J.G.: Mathematical Models in the Social Sciences, New York. (1962)

12. Kendall, M.G.: A new measure of rank correlation. Biometrika. 30, 81--93 (1938)

13. Bansal, M, Fernández-Baca, D.: Computing distances between partial rankings. Information Processing Letters. 109, 238--241 (2009)

14. Hausdorff, F.: Grundzüge der Mengenlehre, Leipzig (1914)

15. Fagin, R., Kumar, R., Mahdian, M., Sivakumar, D., Vee, E.: Comparing partial rankings. Journal of Discrete Mathematics. 20, 628--648 (2006)

16. Dorigo, M., Caro, G.D., Gambardella, L.: Ant algorithms for discrete optimization. Artificial Life. 5, 137--172 (1999)

17. Dorigo, M., Bonabeau, E., Theraulaz, G.: Ant algorithms and stigmergy. Future Generation Computer Systems. 16, 851--871 (2000)

18. Dorigo, M., Colorni, A., Maniezzo, V.: The ant system: Optimization by a colony of cooperating agents. IEEE Transactions on Systems and Cybernetics. 26, 29--41 (1996)

19. Dorigo, M., \& Gambardella, L.: Ant colony system: A cooperative learning approach to the traveling salesman problem. IEEE Trans. on Evolutionary Computation. 1, 53--66 (1997)

20. Stützle, T., Hoos, H. H.: MAX-MIN ant system. Future Generation Computer System. 16, 889--914 (2000)

21. Friedman, M.: The use of ranks to avoid the assumption of normality implicit in the analysis of variance. Jour. of the American Statistical Association. 32, 674--701 (1937)

22. Wilcoxon, F.: Individual comparisons by ranking methods. Biometrics. 1, 80--83 (1945) 